\title{
AD-HOC STUDY OF PHARMA COMPANIES' WILLINGNESS TO CONDUCT PASS/PAES STUDIES IN BULGARIA
}

\author{
Polina Paparkova, Maria Dimitrova, Violeta Getova, Ilko Getov \\ Faculty of Pharmacy, Medical University - Sofia
}

\begin{abstract}
INTRODUCTION: According to contemporary data very small part of the developed and clinically tested molecules reach the market. Before being authorized for human use, during the clinical trials, the medicinal product is tested in many different ways. Bulgaria is one of the most preferred countries for conducting clinical trials. According to the current regulation the post-authorization studies are divided into two types - PASS (safety studies) and PAES (studies of efficacy) - also known as phase 4 of the clinical trials, taking place in the real clinical practice with patients. Their aim is identification, quantifying and assessment of the importance of the risks, linked to the use of a medicinal product in the clinical practice.

MATERIALS AND METHODS: A questionnaire composed with the aim to assess and compare the willingness of the pharmaceutical companies in Bulgaria to conduct PASS/PAES before and after the implementation of the new pharmacovigilance legislation in 2012 has been carried out. Subjects of the study were pharmaceutical companies and representatives of marketing authorization holders (MAH) in Bulgaria - innovative companies, members of the Association of the Research-Based Pharmaceutical Manufacturers in Bulgaria (ARPharM) and generic companies, members of the Bulgarian Generic Pharmaceutical Association (BgPharmA).

RESULTS: Six of the innovative and none of the generic companies declare to have conducted post-authorization studies before 2012. Currently in international perspective half of the questioned pharmaceutical companies and two of the generic ones conduct PASS and only one of the innovative companies conduct PAES. Nowadays, only one of the questioned innovative companies has current PASS and PAES in Bulgaria.

CONCLUSION: The results of the conducted study show that from regulatory and logistic point of view in Bulgaria all the required conditions for conduction of PASS and PAES are present. Although this legal tool is not yet widely popularized in the activities of the regulatory authority and MAHs, the existing conditions have the potential to speed up the development of this sector of the pharmaceutical industry.
\end{abstract}

Keywords: medicinal product, PASS, PAES, clinical trial

Address for correspondence:

Violeta Getova

Department of Organization and Economics of Pharmacy

Faculty of Pharmacy

Medical University - Sofia

2 Dunav Str.

1000 Sofia

e-mail:violeta.getova@bda.bg

Received: April 14, 2016

Accepted: September 30, 2016

\section{INTRODUCTION}

According to contemporary data very limited number of the developed and clinically tested molecules reaches the market. When a particular therapeutic effect of a tested molecule is detected, this molecule can be authorized for human use after series of laboratory testing on animal species (a.k.a. pre-clinical phase), conducted according to the Good Laboratory Practice. After this stage is successfully final- 
Polina Paparkova, Maria Dimitrova, Violeta Getova et al.

ized, the testing for human use in the phases of the clinical trials begins. The analyses show that from 50000 newly synthesized molecules five are used in humans and only one of them turns out to be efficacious and safe enough to receive a marketing authorization. During these tests it is being determined whether the therapeutic efficacy and safety are comparable to these of other medicinal products that are already implemented in the practice. According to statistical data only one out of every 12 molecules that go through clinical trials actually reaches the pharmaceutical market. Having the most favorable conditions, the whole process from the beginning of the trials until the introduction of the product on to the market takes between seven and nine years and its average price is between 600 million and one billion euro without taxes and manufacturing and delivery expenses. At the same time, the effectiveness of the research and development activities (measured as expense for a successfully introduced molecule) is constantly diminishing. That is why the sponsors are currently looking for ways to optimize this section of the pharmaceutical industry.

Data of the National Institute of Health USA from 2013 shows that 106649 clinical trials are being conducted in 174 countries worldwide, 27093 of which are held in Europe.

Lately, Bulgaria has been one of the highly preferred countries for conduction of clinical trials. According to the European Medicines Agency (EMA) database of clinical trials in Europe, nowadays (2016) 851 out of 13530 trials are conducted in Bulgaria which is around $6 \%$ of the total number in Europe (1). One of the motives is that Bulgaria has a lower level of quality of health services compared to the developed countries. It is considered that the participation in clinical trials can assure higher quality of treatment and access to innovative medicines. The investment of the international pharmaceutical companies for conduction of clinical trials in Bulgaria is estimated to be around 200-500 million Bulgarian leva a year, approx. $102-255$ million $€$. The analysis conducted by KPMG shows that the clinical trials sector brings to the Bulgarian economics 77.5 million $€$ per year as expenses for salaries, repayment for the hospitals and the exploiters, legal taxes, etc. (2).
According to the latest changes in the clinical trials regulation the post-authorization studies are two types - PASS (safety studies) and PAES (studies of efficacy). This stage is known as phase 4 of the clinical trials and takes place in the real clinical practice with patients.

Post-authorization studies can be conducted when the medicinal product is being used according to the approved summary of product characteristics $/ \mathrm{SmPC} /$ in the post-authorization period. Their aim is identification, quantity assessment and assessment of the importance of the risks, linked to the use of a medicinal product in the clinical practice. PASS conduction is legally framed in Directive 2001/83 (3).

This type of studies is particularly suitable for obtaining new information on the prognosis, duration, and quality of life in actual conditions. The frequency of both known and unknown ADRs is being studied. PASS also gives opportunity for research of new indications of the medicinal product as well as new dose regimens. Another advantage of PASS is the possibility to detect, find and analyze eventual risks in special population groups (4).

\section{MATERIALS AND METHODS}

A questionnaire composed with the aim to assess the willingness of the pharmaceutical companies in Bulgaria to conduct PASS/PAES has been carried out. It describes the period before the implementation of the pharmacovigilance legislation in June, 2012 and shows how the tendencies in the willingness of companies have changed with the new legal requirements in the pharmacovigilance field. The questionnaire begins with short appeal to the public to point out the subject and the aim of the survey, who conducts it and how the collected data will be used. The questions are presented in a funnel-shaped way beginning with general questions and going more concrete to the adopted practices for conducting PASS and PAES. The questionnaires are divided depending on the targeted respondents - questionnaires conducted with innovative companies and questionnaires conducted with generic companies. It consists of six questions. The received answers sketch the basic frame of the obligations of the companies to conduct post-authorization studies for safety and efficacy internationally and on a local basis as well as the companies' willingness to do it. 
The questionnaires are structured so that the results will be easy to code, process and analyze. Descriptive statistics were applied using SPSS software. The low responding rate and small sample size does not allow a comparison between the two groups.

Subjects of the study were pharmaceutical companies and representatives of marketing authorization holders (MAH) in Bulgaria - innovative companies, members of the Association of the ResearchBased Pharmaceutical Manufacturers in Bulgaria (ARPharM) and generic companies, members of the Bulgarian Generic Pharmaceutical Association (BgPharmA). 16 companies participated in the survey - 10 innovative and 6 generic - for a period of three months in 2015.

The questions are divided into three categories - requirements for conduction of PASS and PAES before 2012, conduction of PASS and PAES after 2012 internationally and in Bulgaria and importance and influence of the results on the SmPCs.

The analysis of the results includes the requirements to the companies with the following criteria:

1. Conduction of PASS and PAES.

\section{Participants in the questionnaire}

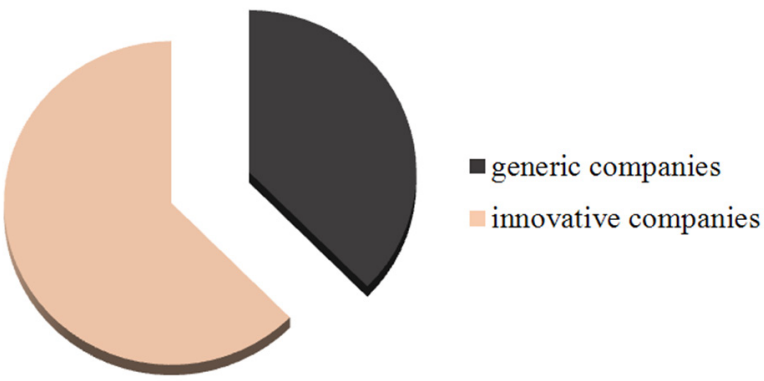

Figure 1. Number of companies which participated in the questionnaire in a three-month period

7. Deadlines for receiving authorization for conducting PASS and PAES.

\section{RESULTS AND DISCUSSION}

The participants in the survey were rather innovative (10) than generic (6) companies.

In the processing of the data the names of the companies were numerically coded from 1 to 10 (for innovative companies) and from 1 to 6 (generic companies).

Table 1. Conduction of PASS and PAES in the pharmaceutical industry before 2012

\begin{tabular}{|c|c|c|c|c|c|c|c|c|c|c|c|c|c|c|c|c|}
\hline & \multicolumn{10}{|c|}{ Innovative companies } & \multicolumn{6}{|c|}{ Generic companies } \\
\hline & 1 & 2 & 3 & 4 & 5 & 6 & 7 & 8 & 9 & 10 & 1 & 2 & 3 & 4 & 5 & 6 \\
\hline $\begin{array}{l}\text { Conduction of } \\
\text { PASS and PAES } \\
\text { before } 2012\end{array}$ & Yes & Yes & No & No & Yes & Yes & Yes & Yes & No & No & No & No & No & No & No & No \\
\hline
\end{tabular}

2. Conduction of PASS and PAES for new medicinal products and for those under additional monitoring.

3. Conduction of PASS and PAES for generic medicinal products.

4. Marking with a black triangle in the SmPCs of all medicinal products which need to be additionally monitored after receiving marketing authorization.

5. Commission for assessment of the risk/benefit ratio of the medicinal products.

6. Requirements for the documentation needed for receiving authorization for conducting PASS and PAES.
Conduction of the PASS and PAES before the changes in the European legislation regarding Pharmacovigilance

Six of the total 10 questioned innovative companies confirm to have conducted phase 4 clinical trials. The biggest part of them was non-interventional, observational, pharmacoepidemiological and studies on records of morbidity.

The questioned generic companies do not report conduction of PASS and PAES before the legislation changes.

Conduction of PASS and PAES Internationally and in Bulgaria after Implementation of the New European Pharmacovigilance 
Polina Paparkova, Maria Dimitrova, Violeta Getova et al.

\section{International Perspective}

Currently in international perspective half of the questioned innovative pharmaceutical companies (5) and two of the generic ones conduct PASS with a total number of studies varying between 1 and 20 studies per company. Only one of the innovative companies conducts PAES. Two of the innovative companies and two of the generic do not report data for this type of studies in international perspective and two of the innovative companies have responded that after 2012 they have conducted such studies but currently they are not active. One of the innovative companies has stated that it is currently conducting PASS but without further information on their number.

\section{Bulgaria}

Nowadays, only one of the questioned innovative companies has current PASS and PAES in Bulgaria.

Pharmaceutical Industry's Attitude Towards the Conditions for Conducting PASS and PAES in Bulgaria and the Validity of the Obtained Data and Results

Four of the generic companies which participated in the survey consider Bulgaria to have good enough conditions for conducting PASS and PAES for collecting valid data. The main factors that influence the process are the type of the medicinal product, morbidity, and the number of patients partici-

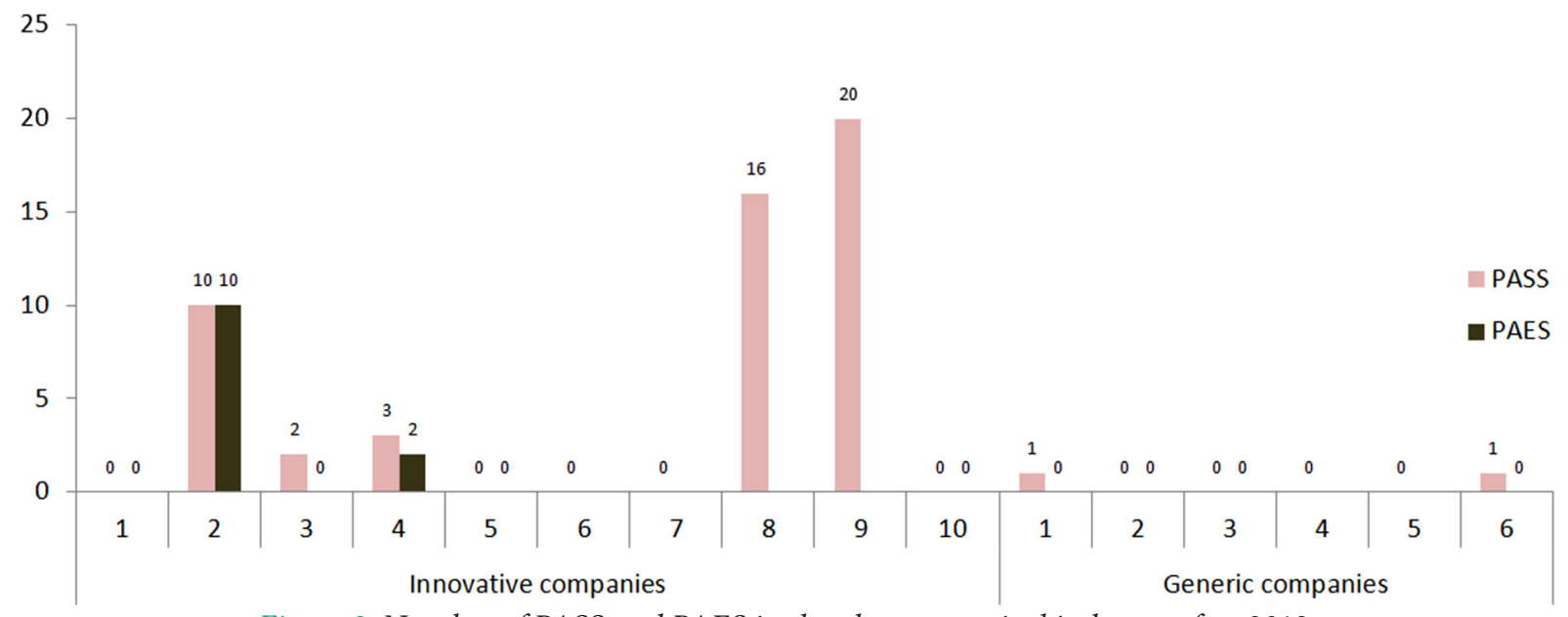

Figure 2. Number of PASS and PAES in the pharmaceutical industry after 2012

Three of the innovative companies share in the questionnaire that EMA or FDA had obliged them to conduct a post-authorization study of efficacy and two of them had to change their SmPCs due to the collected results. This result clearly shows that the implementation of the new legislation in June, 2012 has somehow influenced the pharmaceutical companies and has led them to establish new tools for postmarketing surveillance.

Generic companies are still more passive in the conduction of post-authorization studies compared to the innovative ones. It is also clear that the industry would pursue safety studies rather than studies of efficacy. pating in the study.

All of the innovative companies think that from a regulatory and logistic point of view in Bulgaria all the required conditions for conduction of PASS and PAES are present.

Despite these declarations of opinion it is obvious that in Bulgaria the MAH representatives are not yet familiar and experienced enough with this type of studies. This is one of the reasons for the low number of PASS and PAES.

\section{CONCLUSION}

The conducted study on the importance, knowledge and willingness of the pharmaceutical companies in Bulgaria to conduct PASS and PAES is 
the first one in the country which allows some conclusions and instructions to be made.

The data on the willingness to conduct PASS and PAES show that this legal tool is not yet widely popularized in the activities of the regulator authority and MAHs. The main limiting factors in this sector are related to the restrictive regulatory frame, especially concerning approval of new trials.

We consider it important that MAHs share the opinion that in Bulgaria all the needed conditions for conducting this type of trials are present. Therefore, our country has the potential for future development in this sector from which healthcare professionals, hospitals, academia and regulatory authorities would benefit (5). The ongoing reforms also include an upgrade of the legislative frame for conducting clinical trials, optimization of the number of documents required, expansion of the administration capacity of the Bulgarian Drug Agency and Ethics Commission in order to meet the requirements of Regulation 536/2014, creation of an electronic database which will be maintained by the BDA, enhancement of the interactions between the scientists, industry and administration.

\section{REFERENCES}

1. www.clinicaltrialsregister.eu [accessed September 2016]

2. KPMG Bulgaria OOD - Clinical Trials in Bulgaria - Key challenges, 2015, www.kpmg/bg

3. EMA. Guideline on good pharmacovigilance practices (GVP). GL 27 (2013). doi:EMA/876333/2011 Rev. $1^{\star}$

4. Atanasov V., Kostov E., Lebanova H., Getova V., Popova M., Getov I., Characteristics and nature of non-interventional studies, Science Pharmacology, $1 / 2016$

5. National strategy for development of clinical trials and non-interventional studies in Bulgaria, draft for public discussion, available on www. mh.government.bg, last accessed 10.02.2016

6. EC. Commission Delegated Regulation 357/2014. (2014)

7. EC. Regulation (EU) 536/2014 on clinical trials on medicinal products for human use, and repealing Directive 2001/20/EC 